\title{
Numerical simulations of a fully developed liquid-metal magnetohydrodynamic flow in a circular duct
}

\author{
Cai Xinghui ${ }^{\mathrm{a}}$, Qiang Hongfu, Dong Sanqiang, Wang Guoliang, Lu Jiangren \\ Xi'an Research Inst. of Hi-Tech, Hongqing Town, Xi'an, P. R. China
}

\begin{abstract}
In this paper, a kind of new meshless method, two-level radial point interpolation method, has been developed to analyze the fully developed liquid-metal Magnetohydrodynamic (MHD) flow under the externally applied magnetic field in a circular duct with thin conducting walls. This method applied the radial point interpolation method (RPIM) to solve the two levels Galerkin weak form formulations. As the shape functions from RPIM possess Kronecker delta function properties, the essential boundary conditions of MHD flow problems are easily to be enforced. Numerical simulations of MHD flow in the circular duct with partial conducting walls are carried out, showing that the method is very stable in the computation.
\end{abstract}

\section{Introduction}

The study on the liquid metal Magnetohydrodynamic (MHD) flow in a duct has taken many interests of researchers due to its applications in the cooling system with liquid metals for nuclear fission or fusion reactors, electromagnetic pumps, MHD generators, etc. Since the analytical solutions can be achieved only in some simple cases $^{[1], ~[2], ~ n u m e r i c a l ~ s i m u l a t i o n ~ h a s ~ b e c o m e ~ a ~ v e r y ~}$ important tool for MHD flow problems. The radial point interpolation method (RPIM), employing the radial basis function as its shape function, is a very attractive meshless method proposed ${ }^{[3]}$ and analyzed ${ }^{[4]}$ by JG Wang and GR Liu. The radial point interpolation method was employed by XH Cai et al. ${ }^{[5]}$ to the fully MHD flow problems for Hartmann numbers less than 50. By using the residual-free bubble functions, L Zhang et al. ${ }^{[6]}$ proposed a new meshless method-two level elementfree Galerkin method and applied it to fully developed MHD flow and obtained the numerical results for Hartmann numbers up to $10^{4}$.

Compared with element-free Galerkin method, the shape functions from RPIM possess the Kronecker delta function properties, which make it easier and more accurately to enforce the essential boundary conditions. In this paper, a two-level radial point interpolation method (TLRPIM), which may enforce the essential boundary conditions directly, is proposed and applied to solve the fully developed MHD flow in a circular duct.

\section{The physical problem}

The external applied magnetic field $B_{0}$ is in the $x y$-plane, which is also a cross section of the rectangular duct, but forming an angle $\alpha$ with the $y$-axis. The flow direction is along the $z$-axis, which is also the axial direction of the duct. Then the governing equations for the fully developed MHD flows can be written in nondimensional form ${ }^{[7]}$ as

$$
\begin{aligned}
& \nabla^{2} V+H a_{X} \frac{\partial B}{\partial X}+H a Y \frac{\partial B}{\partial Y}=-1 \\
& \nabla^{2} B+H a_{X} \frac{\partial V}{\partial X}+H a_{Y} \frac{\partial V}{\partial Y}=0
\end{aligned}
$$

in the flow domain $\Omega, \frac{\partial^{2} B^{\prime}}{\partial X^{2}}+\frac{\partial^{2} B^{\prime}}{\partial Y^{2}}=0$ in the duct wall $\Gamma$, where $H a_{X}=H a \sin \alpha, H a_{Y}=H a \cos \alpha, H a=\sqrt{H a_{X}^{2}+H a_{Y}^{2}}$.

If the thickness $h$ of the duct wall is much smaller than the characteristic dimension $a$, the boundary conditions can be written as follows ${ }^{[1]}$ :

$$
V=0, \frac{\partial B}{\partial n}+\theta B=0
$$

where $\theta=\frac{\sigma a}{\sigma^{\prime} h}$.

In the above equations, $\sigma$ and $\sigma^{\prime}$ are the electric conductivity of the fluid and the wall, respectively. $V$ is the non-dimensional velocity of the fluid. $B$ and $B$ ' are the non-dimensional induced magnetic field of the fluid and the duct walls, respectively. $n$ is the unit normal vector to the boundary of the flow section. $\mathrm{Ha}$ is the Hartmann number. The non-dimensional parameters in the above

\footnotetext{
$\bar{a}$ Corresponding author: hui_xing1975@sina.com
} 
equations are $X=\frac{x}{a}, Y=\frac{y}{a}, B=-\frac{B_{z}}{\left(a^{2} / \eta\right)(\partial p / \partial z) \mu_{0} \sqrt{\eta \sigma}}$, $V=-\frac{v_{z}}{\left(a^{2} / \eta\right) \partial p / \partial z}, H a=B_{0} a \sqrt{\frac{\sigma}{\eta}}$. where $\eta$ is the fluid viscosity coefficient. $v_{z}(x, y), B_{z}(x, y)$ and $p$ are the axial velocity, the induced magnetic field and the pressure, respectively. $\mu_{0}$ is a constant, which has the value $4 \pi \times 10^{-7}$ in the MKS system. $a$ is the characteristic length of the flow section.

\section{Numerical method}

\subsection{Radial point interpolation method}

An approximation of a continuous function $V(\mathbf{x})$ defined on a domain $\Omega$ can be obtained through the point interpolation method by using radial basis functions (RBFs) and polynomial basis:

$$
\begin{gathered}
V^{h}(\mathbf{x})=\sum_{i=1}^{n} R_{i}(\mathbf{x}) h_{i}+\sum_{j=1}^{m} p_{j}(\mathbf{x}) q_{j} \\
=\left(\mathbf{R}^{T}(\mathbf{x}) \mathbf{p}^{T}(\mathbf{x})\right)\left(\begin{array}{l}
\mathbf{h} \\
\mathbf{q}
\end{array}\right) \\
R_{i}(\mathbf{x})=R_{i}\left(r_{i}\right)
\end{gathered}
$$

where $R_{i}(\mathbf{x})$ is a radial basis function associated with node $i, p_{j}(\mathbf{x})$ is a monomial in the space coordinates $\mathbf{x}^{T}=[x, y], n$ is the number of nodes in the neighborhood of $\mathbf{x}, m$ is the number of monomial basis functions. $h_{i}$ and $q_{j}$ are the interpolation constants. $r_{i}$ is the distance between the interpolation point $\mathbf{x}$ and the node $\mathbf{x}_{i}$.

In order to solve Eq. (4), a system of linear algebraic equations is given by

$$
\tilde{\mathbf{V}}_{S}=\underbrace{\left[\begin{array}{lr}
\mathbf{R}_{0} & \mathbf{p}_{m} \\
\mathbf{p}_{\mathrm{m}}^{T} & \mathbf{0}
\end{array}\right]}_{\mathbf{G}}\left(\begin{array}{l}
\mathbf{h} \\
\mathbf{q}
\end{array}\right)
$$

with the constraint equations:

$$
\sum_{j=1}^{m} p_{j}\left(\mathbf{x}_{\mathbf{i}}\right) h_{i}=\mathbf{p}_{m}^{T} \mathbf{h}=0 \quad j=1,2, \ldots, m
$$

where

$$
\begin{aligned}
& \tilde{\mathbf{V}}_{S}=\left(\begin{array}{llllllll}
V_{1} & \mathrm{~V}_{2} & \ldots & \mathrm{V}_{n} & 0 & 0 & \ldots & 0
\end{array}\right) \\
& \mathbf{R}_{0}^{T}=\left[\begin{array}{cccc}
R_{1}(\eta) & R_{2}(\eta) & \ldots & R_{n}(\eta) \\
R_{1}\left(r_{2}\right) & R_{2}\left(r_{2}\right) & \ldots & R_{n}\left(r_{2}\right) \\
\ldots & \ldots & \ldots & \ldots \\
R_{1}\left(r_{n}\right) & R_{2}\left(r_{n}\right) & \ldots & R_{n}\left(r_{n}\right)
\end{array}\right]_{n \times n}
\end{aligned}
$$

$$
\mathbf{p}_{m}^{T}=\left[\begin{array}{llll}
1 & 1 & \ldots & 1 \\
x_{1} & x_{2} & \ldots & x_{n} \\
y_{1} & y_{2} & \ldots & y_{n} \\
\ldots & \ldots & \ldots & \ldots \\
p_{m}\left(\mathbf{x}_{1}\right) & p_{m}\left(\mathbf{x}_{2}\right) & \ldots & p_{m}\left(\mathbf{x}_{n}\right)
\end{array}\right]_{m \times n}
$$

Substituting Eq. (6) into Eq. (4) yields:

$$
V^{h}(\mathbf{x})=\left(\mathbf{R}^{T}(\mathbf{x}) \mathbf{p}^{T}(\mathbf{x})\right) \tilde{\mathbf{V}}_{S} \mathbf{G}^{-1}=\tilde{\boldsymbol{\Phi}}^{T}(\mathbf{x}) \tilde{\mathbf{V}}_{S}
$$

where

$$
\begin{aligned}
& \tilde{\boldsymbol{\Phi}}^{T}(\mathbf{x})=\left(\mathbf{R}^{T}(\mathbf{x}) \mathbf{p}^{T}(\mathbf{x})\right) \mathbf{G}^{-1} \\
& =\left(\begin{array}{llllll}
\Phi_{1}(\mathbf{x}) & \Phi_{2}(\mathbf{x}) & \ldots & \Phi_{k}(\mathbf{x}) & \ldots & \Phi_{n+m}(\mathbf{x})
\end{array}\right)
\end{aligned}
$$

are RPIM shape functions. The shape function obtained above possesses the Kronecker delta function properties, i.e.

$$
\Phi_{i}\left(\mathbf{x}_{j}\right)=\delta_{i j}= \begin{cases}1, & i=j \\ 0, & i \neq j\end{cases}
$$

E.J. Kansa ${ }^{[8]}$, M. Sharan ${ }^{[9]}$, C. Frank ${ }^{[10]}$ have deeply investigated on RBFs, such as multiquadrics function, Gaussian (Exp) function, and TPS function, and proposed some constructive suggestions on how to use them. In this paper, multiquadrics RBF is applied, which is defined as

$$
R_{i}(x, y)=\left(r_{i}^{2}+\left(a_{c} d_{c}\right)^{2}\right)^{q}
$$

where $a_{c}$ and $q$ are shape parameters.

\subsection{Two-level weak formulation}

Using the weighted residual method, a weak form of (1) and (2), over $\Omega$ bounded by $\partial \Omega$ can be obtained as:

$$
\begin{gathered}
\int_{\Omega} \nabla V \cdot \nabla \hat{V} d \Omega-\int_{\Omega} H a_{X} \frac{\partial B}{\partial X} \hat{V} d \Omega- \\
\int_{\Omega} H a_{Y} \frac{\partial B}{\partial Y} \hat{V} d \Omega-\int_{\partial \Omega} \hat{V} \nabla V \cdot \mathbf{n} d \Omega=\int_{\Omega} \hat{V} d \Omega \\
\int_{\Omega} \nabla B . \nabla \hat{B} d \Omega-\int_{\Omega} H a_{X} \frac{\partial V}{\partial X} \hat{B} d \Omega- \\
\int_{\Omega} H a Y \frac{\partial V}{\partial Y} \hat{B} d \Omega-\int_{\partial \Omega} \hat{B} \nabla B . \mathbf{n} d \Omega=0
\end{gathered}
$$

Assumed that velocity $V$ and magnetic field $B$ can be decomposed as:

$$
\left\{\begin{array}{l}
V(X, Y)=V_{a}(X, Y)+V_{b}(X, Y) \\
B(X, Y)=B_{a}(X, Y)+B_{b}(X, Y)
\end{array}\right.
$$

where $V_{a}$ and $B_{a}$ represent the solutions obtained by RPIM, $V_{b}$ and $B_{b}$ will be solved by the residual-free bubble functions. Similarly, their corresponding weighting functions $\hat{V}$ and $\hat{B}$ can also be decomposed into the sum of $\hat{V}_{a}(X, Y)$ and $\hat{V}_{b}(X, Y)$, $\hat{B}_{a}(X, Y)$ and $\hat{B}_{b}(X, Y)$, respectively.

In the interior of each background integration cell $\Omega^{\text {cell }}$ of PRIM, the MHD govern equations become 


$$
\begin{cases}\nabla^{2}\left(V_{a}+V_{b}\right)+\text { Ha. } \nabla\left(B_{a}+B_{b}\right)=-1 & \text { in } \Omega^{\text {cell }} \\ \nabla^{2}\left(B_{a}+B_{b}\right)+\text { Ha. } \nabla\left(V_{a}+V_{b}\right)=0 & \text { in } \Omega^{\text {cell }} \\ V_{b}=B_{b}=0 & \text { on } \partial \Omega^{\text {cell }}\end{cases}
$$

$\hat{V}_{b}$ and $\hat{B}_{b}$ are neglected since they are much less than $\hat{V}_{a}$ and $\hat{B}_{a}$ respectively. Substitution of the trial solutions and the weighting functions in the standard variational form yields

$$
\begin{aligned}
& -\left(\nabla V_{a}, \nabla \hat{V}_{a}\right)+\left(\text { Ha. } \nabla B_{a}, \hat{V}_{a}\right)- \\
& \left(\nabla V_{b}, \nabla \hat{V}_{a}\right)+\left(\text { Ha. } \nabla B_{b}, \hat{V}_{a}\right)=\left(-1, \hat{V}_{a}\right) \\
& -\left(\nabla B_{a}, \nabla \hat{B}_{a}\right)+\left(\text { Ha. } \nabla V_{a}, \nabla \hat{B}_{a}\right)- \\
& \left(\nabla B_{b}, \nabla \hat{B}_{a}\right)+\left(\text { Ha. } \nabla V_{b}, \hat{B}_{a}\right)=0
\end{aligned}
$$

RPIM is applied to solve (18) and (19), and bubble functions $\left(V_{b}, B_{b}\right)$ are obtained by RPIM with the adaptive upwind scheme ${ }^{[11]}$.

Let

$$
H_{b}=V_{b}+B_{b}, \quad F_{b}=V_{b}-B_{b}
$$

and then Eq. (17) becomes:

$$
\left\{\begin{aligned}
\nabla^{2} H_{b}+\text { Ha. } \nabla H_{b} & =-1-\left(\nabla^{2} V_{a}+\text { Ha. } \nabla V_{a}\right) \\
& -\left(\nabla^{2} B_{a}+\text { Ha. } \nabla B_{a}\right) \text { in } \Omega^{c e l l} \\
\nabla^{2} F_{b}-\text { Ha. } \nabla F_{b} & =-1-\left(\nabla^{2} V_{a}-\mathbf{H a} \cdot \nabla V_{a}\right) \\
& +\left(\nabla^{2} B_{a}-\mathbf{H a} \cdot \nabla B_{a}\right) \text { in } \Omega^{c e l l} \\
H_{b}=F_{b}=0 & \text { on } \partial \Omega^{c e l l}
\end{aligned}\right.
$$

Assumed that $\phi_{i}^{V}$ 's, $\phi_{i}^{B}$ 's, $\phi_{f}$ 's are the bubble basis functions for $H_{b}, i=1,2, \ldots, n_{V}$, the number of degrees of freedom for the velocity for $\phi$ 's, then the following equations are obtained as

$$
\begin{aligned}
& \left\{\begin{array}{lc}
\nabla^{2} \varphi_{i}^{V}+\mathbf{H a} \cdot \nabla \varphi_{i}^{V}=-\left(\nabla^{2} \psi_{i}^{V}+\mathbf{H a} \cdot \nabla \psi_{i}^{V}\right) & \text { in } \Omega^{\text {cell }} \\
\varphi_{i}^{V}=0 & \text { on } \partial \Omega^{\text {cell }}
\end{array}\right. \\
& \left\{\begin{array}{lc}
\nabla^{2} \varphi_{i}^{B}+\mathbf{H a} \cdot \nabla \varphi_{i}^{B}=-\left(\nabla^{2} \psi_{i}^{B}+\mathbf{H a} \cdot \nabla \psi_{i}^{B}\right) \text { in } \Omega^{\text {cell }} \\
\varphi_{i}^{B}=0 & \text { on } \partial \Omega^{\text {cell }}
\end{array}\right. \\
& \left\{\begin{array}{lc}
\nabla^{2} \varphi_{f}+\mathbf{H a} \cdot \nabla \varphi_{f}=-1 & \text { in } \Omega^{\text {cell }} \\
\varphi_{f}=0 & \text { on } \partial \Omega^{\text {cell }}
\end{array}\right.
\end{aligned}
$$

where $\psi_{i}^{V}$ 's and $\psi_{i}^{B}$ 's are the shape functions of RPIM for $V_{a}$ and $B_{a}$, respectively. The similar set of equations for $F_{b}$ can be obtained by assuming again that $\tilde{\phi}_{i}^{V}$ 's, $\tilde{\phi}_{i}^{B}$ 's, $\tilde{\phi}_{f}$ 's are the bubble basis functions for $F_{b}, i$ $=1,2, \ldots, n_{B}$, the number of degrees of freedom for the magnetic field for $\tilde{\phi}$ 's.

Since $V_{a}=\sum_{i=1}^{n_{v}} V_{i} \psi_{i}^{V}$ and $B_{a}=\sum_{i=1}^{n_{B}} B_{i} \psi_{i}^{B}$, then

$$
\left\{\begin{array}{l}
H_{b}=\sum_{i=1}^{n} V_{i} V_{i} \varphi_{i}^{V}+\sum_{i=1}^{n} B_{i} \varphi_{i}^{B}+\varphi_{f} \\
F_{b}=\sum_{i=1}^{n} V_{i} \tilde{\varphi}_{i}^{V}+\sum_{i=1}^{n} B_{i} \tilde{\varphi}_{i}^{B}+\tilde{\varphi}_{f}
\end{array}\right.
$$

The bubble components $\left(V_{b}, B_{b}\right)$ may be expressed in term of bubbles basis functions:

$$
\left\{\begin{array}{c}
V_{b}=\frac{1}{2}\left[\begin{array}{c}
\sum_{i=1}^{n} V_{i}\left(\varphi_{i}^{V}+\tilde{\varphi}_{i}^{V}\right)+{ }_{i=1}^{\sum_{B}^{B} B_{i}\left(\varphi_{i}^{B}+\tilde{\varphi}_{i}^{B}\right)}+\left(\varphi_{f}+\tilde{\varphi}_{f}\right)
\end{array}\right] \\
B_{b}=\frac{1}{2}\left[\sum_{i=1}^{n} V_{i}\left(\varphi_{i}^{V}-\tilde{\varphi}_{i}^{V}\right)+{ }_{+\left(\varphi_{f}-\tilde{\varphi}_{f}\right)}^{\sum_{i=1}^{n} B_{i}\left(\varphi_{i}^{B}-\tilde{\varphi}_{i}^{B}\right)}\right]
\end{array}\right.
$$

Once the solutions of $\phi_{i}^{V}$ 's, $\phi_{i}^{B}$ 's, $\phi_{f}$ 's, $\tilde{\phi}_{i}^{V}$ 's, $\tilde{\phi}_{i}^{B}$ 's, $\tilde{\phi}_{f}$ 's are obtained, $\left(V_{b}, B_{b}\right)$ representing by (26) are plugged into the global formulation (18) and (19), and then finally the global problem is numerically solved.

\section{Numerical simulations and analyses}

Numerical simulations of the circular duct with partial conducting walls are presented in Figure 1 and 2 for Hartmann number 500 and 1000 , respectively. For the circular duct, the walls are conducting in the section of $|X| \geq 0.5$. The external applied magnetic field is parallel to the $X$-axis.

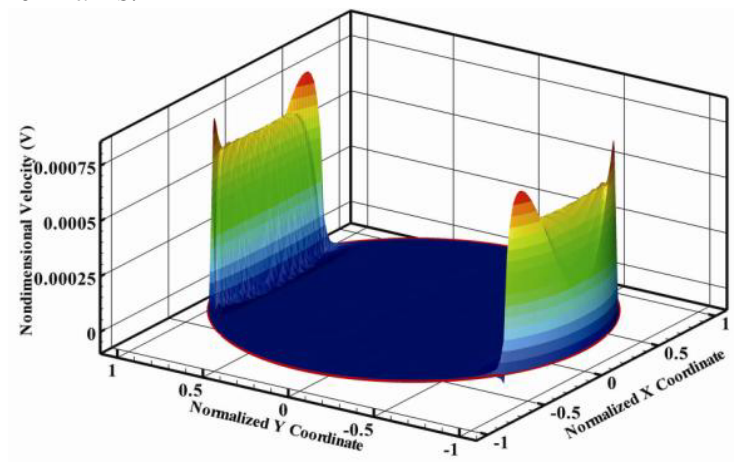

Figure 1. Velocity profile for $H a=500, \theta=10, \alpha=\pi / 2$

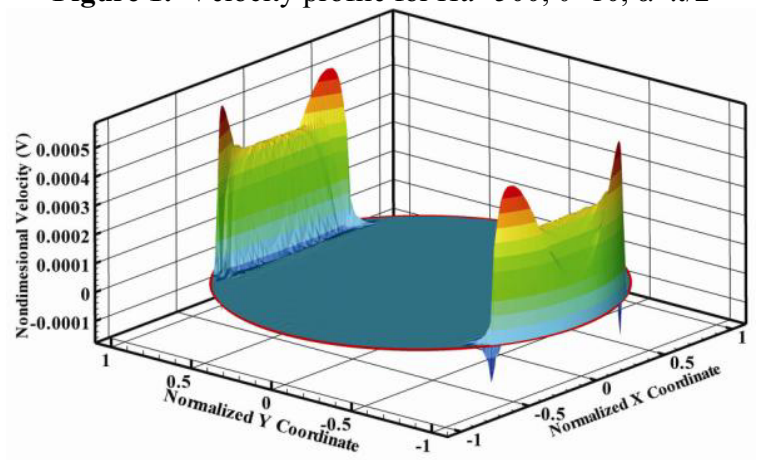

Figure 2. Velocity profile for $H a=1000, \theta=10, \alpha=\pi / 2$

Since there are no analytical solutions could be as comparisons to numerical solutions for MHD flow in circular pipe case, numerical simulation results in Figure. 1 and 2 show a very stable velocity profiles. It is seen that the side layers appear as that in the rectangular duct 
case $^{[8]}$. Different from that the kind of hemicycle shape in the side layers, the velocity profiles in the side layer of the circular pipe show a kind of saddle shape, that is, the largest velocity appeared in the two sides of the side layers, not in the middle part as that in the rectangle duct.

\section{Conclusions}

A two-level radial point interpolation method has been proposed and applied for the fully developed MHD flow in a circular duct. Numerical simulations for MHD flows in circular pipe with Hartmann number 500 and 1000 were performed with good stability.

\section{Acknowledgments}

This work was financially supported by the China Postdoctoral Science Foundation (2014M562635) and the Shanxi Natural Science Foundation (2013JM1005).

\section{References}

1. J.C. HUNT. Magnetohydrodynamic flow in rectangular ducts. J. Fluid Mech 21, 577 (1965)

2. J.A. Shercliff. Steady motion of conducting fluids in pipes under transverse magnetic fields. Proc.Camb.Phil. Soc. 49, 136 (1953)

3. JG Wang, GR Liu. A point interpolation meshless method based on radial basis functions. Int. J. Numer. Meth. Eng. 54, 1623 (2002)

4. JG Wang, GR Liu. On the optimal shape parameters of radial basis functions used for 2-D meshless methods. Comput. Method Appl. M. 191, 2611 (2002)

5. XH Cai, GH Su, SZ Qiu. Local radial point interpolation method for the fully developed Magnetohydrodynamic flow. Appl. Math. Comput. 217, 4529 (2011)

6. L Zhang, OY Jie, XH Zhang. The two-level element free Galerkin method for MHD flow at high Hartmann numbers. Phys. Lett. A 372, 5625 (2008)

7. S.L.L. Verardi, JR Cardoso, CC Motta. A solution of two-dimensional Magnetohydrodynamic flow using the finite element method. IEEE Trans. on Magn. 34, 3134 (1998)

8. E.J. Kansa. Multiquadrics-A scattered data approximation scheme with application to computational fluid dynamics. Comput Math. Appl. 19, 147 (1990)

9. M. Sharan, E.J. Kansa, S Gupta. Application of the multiquadric method for numerical solution of elliptic partial differential equations. Appl. Math. Comput. 84, 275 (1997)

10. C. Frank, R. Schaback. Solving partial differential equations by collocation using radial basis functions. Appl. Math. Comput. 93, 73 (1998)

11. XH Zhang, J Ouyang, JY Wang. Stabilization meshless method for convection dominated problems. Appl. Math. Mech. 29, 1067 (2008) 